\title{
Control strategies for 2-quadrant converter used in grid power flow control
}

Sebastián Maestri, Rogelio Garcia Retegui, Gustavo Uicich, Mario Benedetti, UNIVERSIDAD NACIONAL DE MAR DEL PLATA L.I.C, Mar del Plata, Argentina Konstantinos Papastergiou, Gilles Le Godec

CERN, Geneva, Switzerland,

Keywords: $<<$ Transfer Lines $>>$, $<<$ Energy recovery $>>$, $<<$ Magnet supply $>>,<<$ Control strategy $>>$, $<<$ Grid power limitation $>>,<<$ Energy management $>>$

\begin{abstract}
This work presents the analysis of a two-quadrant regulator connected to the DC-link of a 4quadrant magnet supply. The key objective is to present some regulation strategies for controlling the peak power required from the power network as well as to recover the magnet energy into capacitor banks. A comparative study that highlights the trade o_between the size of reactive elements, and the peak current drawn from the electrical network is presented.
\end{abstract}

Presented at: EPE 2015, 7-10 September 2015, Geneva, Switzerland

Geneva, Switzerland

October, 2015 


\title{
Control strategies for 2-quadrant converter used in grid power flow control
}

\author{
†Sebastián Maestri, ${ }^{\dagger}$ Rogelio Garcia Retegui, ${ }^{\dagger}$ Gustavo Uicich, ${ }^{\dagger}$ Mario Benedetti, \\ ${ }^{\ddagger}$ Konstantinos Papastergiou and ${ }^{\ddagger}$ Gilles LeGodec. \\ $\dagger$ UNIVERSIDAD NACIONAL \\ DE MAR DEL PLATA \\ L.I.C. \\ Juan B.Justo 4302 \\ Mar del Plata, Argentina \\ Phone: +54 2234816600 \\ Fax: +542234810046 \\ Email: somaestri@fi.mdp.edu.ar \\ URL: http://www3.fi.mdp.edu.ar \\ $\ddagger$ EUROPEAN ORGANIZATION FOR \\ NUCLEAR RESEARCH - C.E.R.N. \\ TE-EPC GROUP \\ 1211 Geneva 23 \\ Geneva, Switzerland \\ Phone: +41227675549 \\ Fax: +4122766 8301 \\ Email:k.papastergiou@cern.ch \\ URL: http://www.cern.ch
}

\section{Acknowledgments}

This work has been performed under a research contract between the European Organization for Nuclear Research (CERN) Switzerland and the Consejo Nacional de Investigaciones Científicas y Técnicas (CONICET), with the support of the European Particle Physics Latin American Network (EPLANET), the Universidad Nacional de Mar del Plata (UNMDP), the Ministerio de Ciencia, Tecnología e Innovación Productiva (MINCYT), and the Agencia Nacional de Promoción Científica y Tecnológica (ANPCYT).

\section{Keywords}

$<<$ Transfer Lines $>>,<<$ Energy recovery $>>,<<$ Magnet supply $>>,<<$ Control strategy $>>$, $<<$ Grid power limitation $>>,<<$ Energy management $>>$.

\begin{abstract}
This work presents the analysis of a two-quadrant regulator connected to the DC-link of a 4-quadrant magnet supply. The key objective is to present some regulation strategies for controlling the peak power required from the power network as well as to recover the magnet energy into capacitor banks. A comparative study that highlights the trade off between the size of reactive elements, and the peak current drawn from the electrical network is presented.
\end{abstract}

\section{Introduction}

The European Organization for Nuclear Research (CERN) operates more than 5000 power converters supplying power to magnets and other equipment in the accelerator complex. Some of them have been in operation for over 40 years; hence, a consolidation programme has been put in place to ensure high performance reliable operation. These converters, which are usually based on thyristor rectifiers, operate in DC current and are permanently taking energy from the public mains network. Since these converters are reaching their expected lifetime, CERN plans to replace them with modern switching power converters. In the framework of the Transfer line (TT2) Consolidation Programme, a number of studies on energy cycling have been commissioned. The converter must allow the control of the active power flow as well as the recovery of the magnet energy on a cycle-by-cycle basis. Moreover, these topologies are intended to work in pulsed operation with a system of energy recovery, leading to an inherent reduction in the power consumption. 
Table I shows the values of the load and pulse current parameters, where $I_{R E F}$ is the flat-top current reference, $t_{r}$ is the rise time, $t_{f}$ is the fall time, $t_{f t}$ is the flat-top duration and $T_{p}$ is the cycle repetition period. $L_{m}$ and $R_{m}$ are the inductance and resistance magnet, respectively. The application demands the generation of a pulse with a short term and long term minimum precision of $10 \mathrm{ppm}$.

This article presents the analysis of a topology that uses a 2-quadrant converter to link the local energy storage element with the magnet, as shown in Fig. 1. The input stage is composed by an uncontrolled rectifier, connected to an LC input filter, by means of which a stable voltage $v_{d c}$ is obtained. Such voltage represents the DC-bus for the H-bridge, which is used to regulate the current on the magnet. The topology presents a two-quadrant converter (named 2Q), which is connected to the DC-bus in parallel with the H-bridge. The aim of the $2 \mathrm{Q}$ converter is to supply energy to the magnet in order to bind the energy coming from the electrical network to a limit and to store the energy that returns from the magnet during the fall time. The energy provided by the $2 \mathrm{Q}$ converter comes from a capacitor bank $C_{s t}$, which is used as a storage unit.

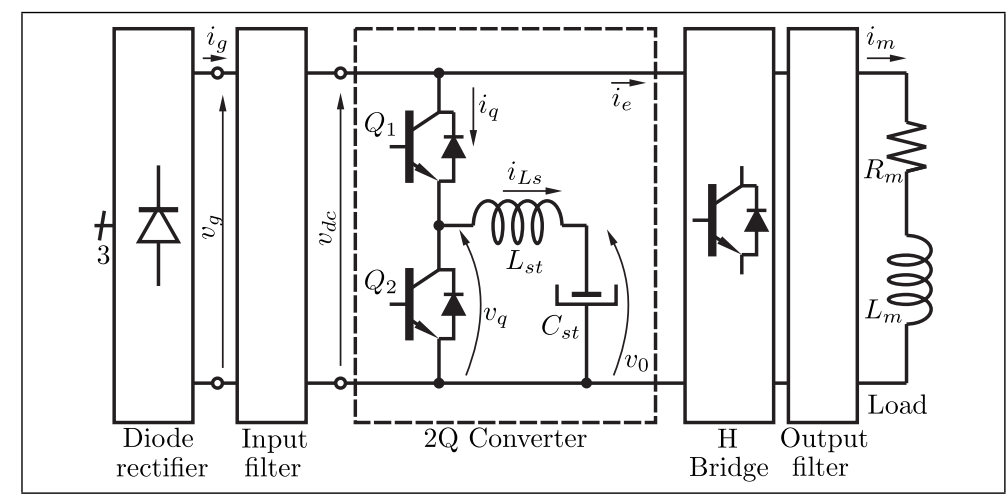

Figure 1: Circuit of 2Q topology.

This topology requires the implementation of different regulation loops, which can be separated in the control of magnet current and the control of power flow between the load and the electrical grid. In the case of the magnet current control, an inner voltage loop and an outer current loop are usually implemented to command the H-bridge stage. The regulation of the H-bridge is out of the scope of this article, which focuses on the study of the regulation strategies to control the flow of energy between the electrical network and the load. Additionally, an electrical network without disturbances will be considered. The effect of grid disturbances in the performance of the system is evaluated in [1].

Concerning the analysis of the energy regulation, two approaches are presented. The first control approach, named Instantaneous Reactive Energy Control (IREC), results in an indirect control of the grid peak power by means of an energy flow balance between the magnet and the energy storing elements using the magnet current information. In some cases, it could happen that this information is not available. This leads to a second approach, named Grid Peak Power Limitation (GPPL), where the reference of the control system is defined by measuring auxiliary local variables, with the aim of bounding the grid current during either generation of pulse or between pulses. In this case, the current demanded by the magnet acts like a perturbation that must be rejected by the control system in order to ensure that the grid current is bounded. In this article, a comparative study between different strategies based on these two approaches is performed.

Table I: Load and pulse parameters (equivalent for one converter module).

\begin{tabular}{c|c|c|c|c|c}
\hline \multicolumn{3}{c|}{ Pulse parameters } & \multicolumn{2}{c}{ Load parameters } \\
\hline$I_{R E F}$ & $450 \mathrm{~A}$ & $t_{f t}$ & $50 \mathrm{~ms}$ & $L_{m}$ & $260 \mathrm{mH}$ \\
\hline$t_{r}$ & $340 \mathrm{~ms}$ & $T_{p}$ & $1.2 \mathrm{~s}$ & $R_{m}$ & $400 \mathrm{~m} \Omega$ \\
\hline$t_{f}$ & $220 \mathrm{~ms}$ & & & & \\
\hline
\end{tabular}




\section{Converter model description}

In order to analyze the topology, it is necessary to find a mathematical model that represents their operation. In this case, it is intended to find a small-signal model from the use of the averaged model concept.

\section{Approximation of $\mathbf{H}$-bridge as a controlled current source}

As a first stage of the modelling, the system is simplified by considering that the magnet current regulation loop (i.e. the H-bridge converter, output filter and magnet) can be modelled as a current source, $i_{e}$, which allows to make the analysis independent of the magnet current regulation. Then, $i_{e}$ is generated from the load power and the input voltage of the $\mathrm{H}$ bridge by considering maximum efficiency, as shown in (1).

$$
v_{d c}(t) \cdot i_{e}(t)=v_{m}(t) \cdot i_{m}(t) \rightarrow i_{e}(t)=\frac{v_{m}(t) \cdot i_{m}(t)}{v_{d c}(t)}
$$

Additionally, a proper design of the input filter avoids that the H-bridge input current affects the voltage $v_{d c}$ and, hence, avoids stability issues $[2,3,4]$. Then, the simplified circuit of the Fig. 2, without the input filter, can be used. In this figure $i_{g}$ is the grid current, $i_{q}$ is the current of the $2 Q$ converter, $i_{L s}$ is the inductor current, $v_{g}$ is the grid voltage and $v_{o}$ is the storage capacitor voltage.

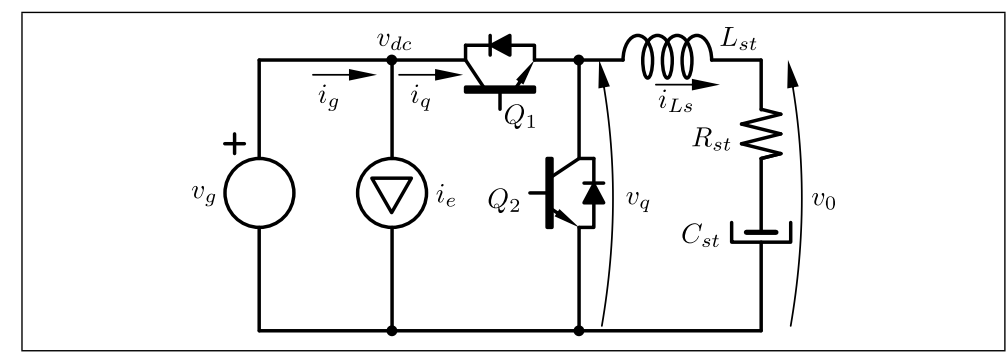

Figure 2: Simplified circuit of 2Q topology.

\section{Averaged model of simplified circuit}

Under the presented conditions, the averaged model of the system shown in Fig. 2 is generated. Such model is obtained as a first order approximation in an operating point. In this sense, the average behavior of a generic variable $x$ is defined as $x=X+\tilde{x}$, where $X$ is the operating point value and $\tilde{x}$ represents the variations of $x$ respect of such operating point $[5,6]$. The equations that define this converter are shown in Table II.

Table II: Average model equations.

\begin{tabular}{l|l}
\hline$i_{g}=i_{e}+i_{q}$ & $i_{g}=I_{G}+\widetilde{i}_{g}, i_{e}=I_{E}+\widetilde{i}_{e}, i_{q}=I_{Q}+\widetilde{i}_{q}$ \\
\hline$i_{q}=d \cdot i_{L s}$ & $d=D+\widetilde{d}, i_{L s}=I_{L s}+\widetilde{i}_{L s}$ \\
\hline$v_{q}=d \cdot v_{g}$ & $v_{q}=V_{Q}+\widetilde{v}_{q}, v_{g}=V_{G}+\widetilde{v}_{g}$ \\
\hline$v_{q}=L_{s t} \frac{d i_{L s}}{d t}+v_{o}$ & $v_{o}=V_{O}+\widetilde{v}_{o}$ \\
\hline
\end{tabular}

The application of the averaged model concept implies solving the equations presented in Table II. In such sense, the system variables are substituted using the representation $x=X+\tilde{x}$ and the second order terms arising from the products of ac terms are eliminated. This process 
leads to the small-signal model presented in Fig. 3(a). Then, a block diagram of the system is obtained (Fig. 3(b)), which will be useful to regulation loops design. Finally, Fig. 4 shows a complete model of the system including the LC input filter stage, where $C_{d c}$ and $L_{d c}$ are the capacitor and inductor of such filter, respectively.

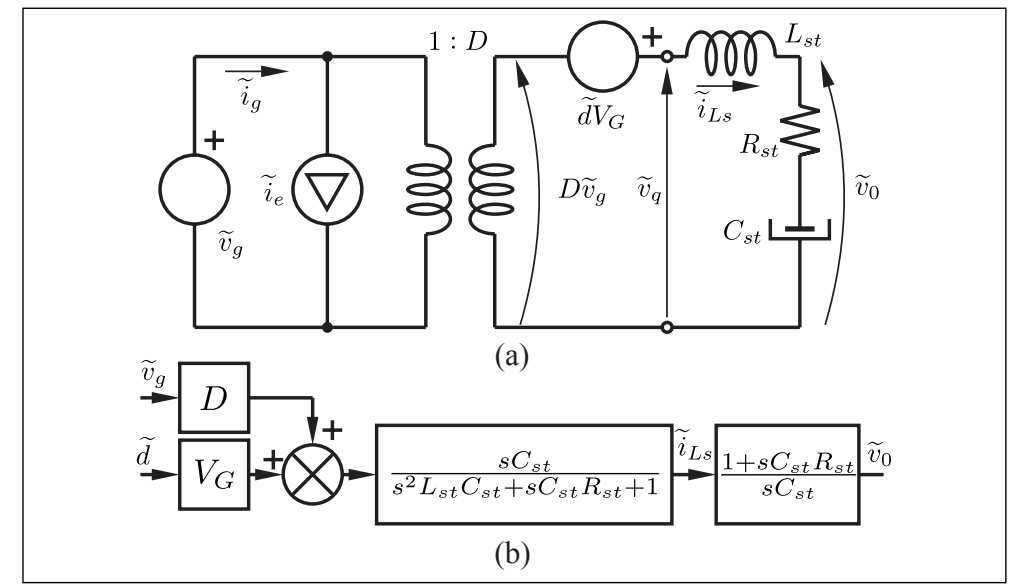

Figure 3: Average-model. (a) Equivalent circuit. (b) Block diagram.

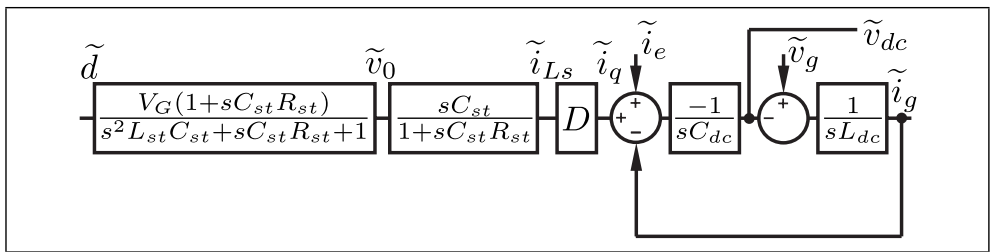

Figure 4: Block diagram of the averaged model.

\section{Control strategies}

The energy flow between the grid and the magnet in this topology is performed by regulating the voltage in the storage element $C_{s t}$. In this sense, the approach named Instantaneous Reactive Energy Control (IREC) makes use of the information of the magnet current $\left(i_{m}\right)$ to obtain the reference for the voltage in this storage element, $v_{r e f}$. In the approach named Grid Peak Power Limitation (GPPL), due to the lack of information of the magnet current, $v_{r e f}$ should be defined from auxiliary local variables, like the grid current $i_{g}$ and/or capacitor filter voltage $v_{d c}$. In the following, a brief description of some strategies based on these approaches is performed.

\section{Control strategy for Instantaneous Reactive Energy Control approach (IREC)}

\section{Strategy: Energy Balance (EB)}

The simplest way to indirectly control the grid power without measuring it, is by ensuring that the reactive power consumed/supplied by the magnet is entirely supplied/recovered by the 2-quadrant converter. This strategy, named Energy Balance, is governed by the following equation:

$$
\frac{1}{2} C_{s t}\left(v_{s t}^{2}\left(t_{0}\right)-v_{s t}^{2}(t)\right)=\frac{1}{2} L_{m} i_{m}^{2}(t)
$$


where $v_{s t}(t)$ is the voltage on $C_{s t}, i_{m}(t)$ is the magnet current, $v_{s t}\left(t_{0}\right)$ is the voltage in $C_{s t}$ when the magnet current is null. In case of having $i_{m}(t)$, this energy balance allows to define the voltage variation in $C_{s t}$, in order to use it as a reference for a closed-loop control of $v_{s t}(t)$. Then, this voltage reference, $v_{r e f}$, is given by (3). By obtaining $v_{r e f}$ from (3), it is assummed that the $2 \mathrm{Q}$ converter must supply the instantaneous reactive energy of the magnet, while the grid must provide the instantaneous active energy.

$$
v_{r e f}(t)=\sqrt{v_{s t}^{2}\left(t_{0}\right)-\frac{L_{m}}{C_{s t}} i_{m}(t)^{2}}
$$

The regulation loops comprise an inner state-feedback regulation and an outer voltage loop, as shown in Fig. 5. The inner state-feedback loop is used to reallocate the poles of the second order system to a frequency, $\omega_{p}$, higher than the desired cut-off frequency. By doing so, it is possible to obtain a bandwidth and phase margin with low dependency on the converter parameters. Concerning the outer loop, an acceptable bandwidth and phase margin is obtained with a PI controller. Using this control scheme, the relationship between $v_{s t}$ and $v_{r e f}$ is given by:

$$
\frac{V_{s t}(s)}{V_{r e f}(s)}=T L C_{v}(s)=\frac{\left(1 / H_{v}\right)\left(1+s C_{s t} R_{s t}\right)}{\left(1+s / \omega_{p}\right)^{2}}
$$

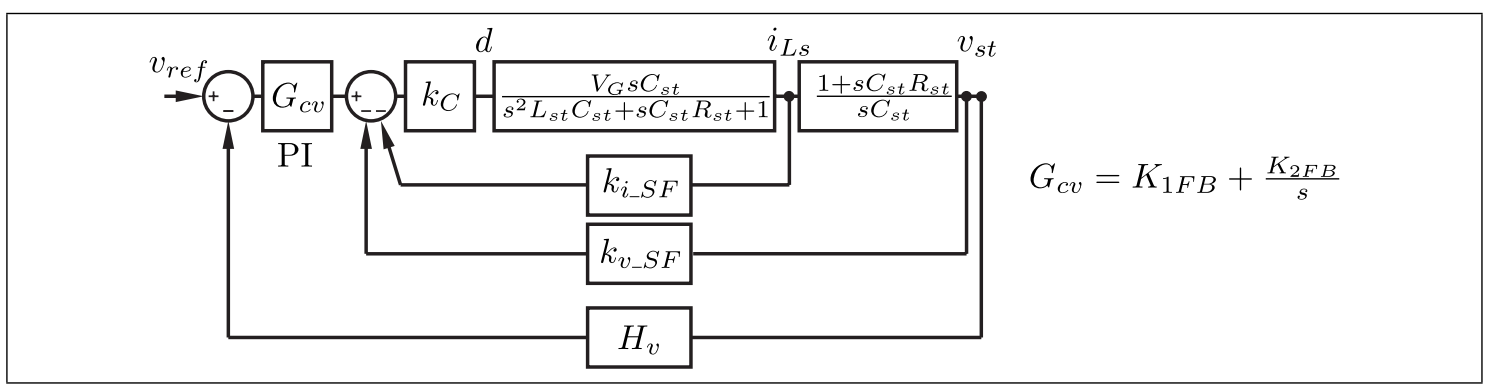

Figure 5: Block diagram of EB control strategy.

\section{Control strategies for Grid Peak Power Limitation approach (GPPL)}

As it was previously mentioned, in the cases where $i_{m}(t)$ is not available, the measurement of $i_{g}$ and/or $v_{d c}$ could be used to obtain $v_{r e f}$ from feedback loops. Then, two control strategies that use this methodology are presented. It must be noticed that, in this case, $i_{e}(t)$ acts like a perturbation for the regulation loops; due to the parabolic shape of this current, the controllers associated to these regulation loops should have into consideration, besides stability issues, the mitigation of the effect of $i_{e}(t)$ in the variables to be controlled.

\section{Strategy: Grid Conduction restricted Between magnet Pulses (GCBP)}

When the variables $i_{g}(t)$ and/or $v_{d c}(t)$ are used for control purposes, the dynamics of the input filter will be included inside the regulation loops. Then, the oscillatory transient response of the LC input filter could become complex the design of the controller. In order to avoid these issues, this strategy proposes to regulate $v_{d c}$ to a value higher than the maximum grid voltage, $\hat{v}_{g}$, during the pulse generation, which prevents the grid to deliver current in this stage by blocking the diodes of the input stage. Hence, all the energy required by the magnet must come from $C_{s t}$, including power losses. Then, after the pulse generation, $C_{s t}$ must restore its initial energy by drawing current from the grid between pulses. This strategy allows splitting the problem in two, according to the task to be performed: 1) delivering of the current required by the magnet and 2) energy recovery of $C_{s t}$. Figure 6 shows a general block diagram of the developed control system, where it can be observed the loop that regulates the voltage $v_{d c}$, which acts during magnet pulse regulation, and the loop that restores the energy at $C_{s t}$ between pulses. 


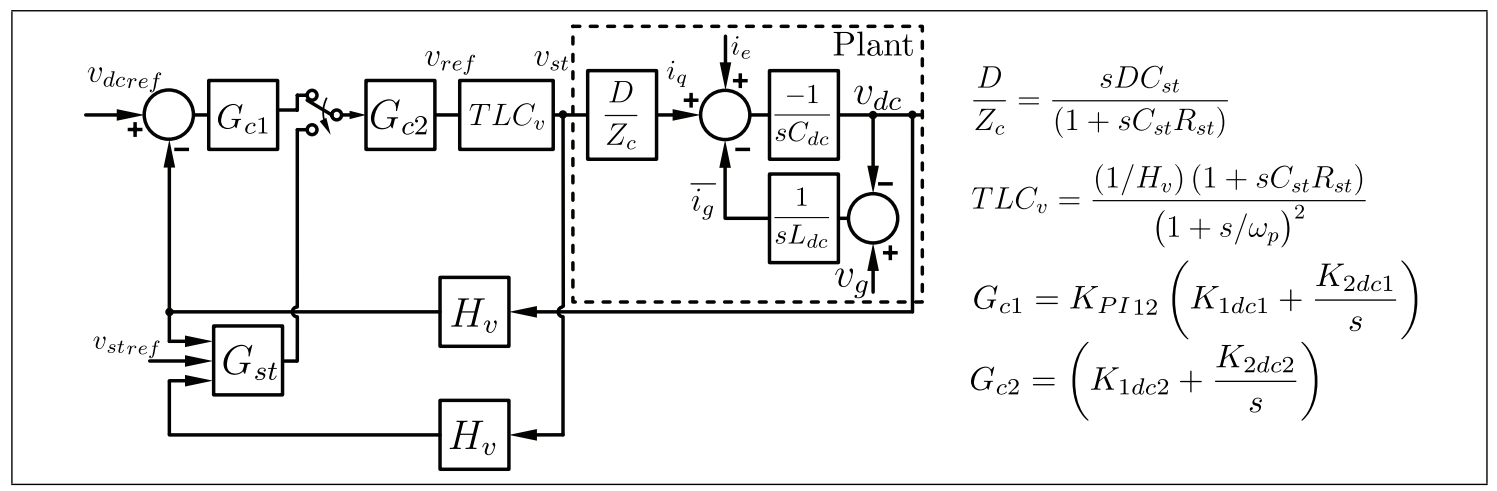

Figure 6: Block diagram of control system for strategy GCBP.

During magnet pulse generation, the regulation loop for $v_{d c}$ is active. The transfer functions in this stage is given by:

$$
G H(s)=G_{c_{1}}(s) G_{c_{2}}(s)\left(-\frac{C_{s t}}{C_{d c}}\right) \frac{D}{\left(1+s / \omega_{p}\right)^{2}}
$$

$G_{c_{1}}$ and $G_{c_{2}}$ are implemented as a double cascaded PI controller, to obtain a small error in $v_{d c}(t)$ due to $i_{e}(t)$. The controller gains of both PI are adjusted to operate with maximum bandwidth and an acceptable phase margin.

When the current demanded by the magnet ends, $i_{e}(t)=0$, the energy recovery of $C_{s t}$ starts. This condition is detected by mean $G_{s t}$ block. The input of $G_{c_{2}}$ is changed to a system that generates the required control signals for obtaining a linear increase/decrease in $v_{r e f}$ (which is equivalent to a positive/negative constant current in $C_{s t}$ ). The change is performed at the input of $G_{c_{2}}$ as it allows generating the initial condition in this controller for the next pulse in a controlled way. The maximum value that has to reach $v_{s t}$, is obtained by taking into account the total energy in $C_{s t}$ and $C_{d c}$ at the beginning and end of the pulse.

\section{Strategy: Indirect Grid Current Control by dc-link voltage regulation (IGCC)}

This strategy is based on the regulation of voltage $v_{d c}$ to obtain a constant current from the grid during the whole period $T_{p}$. In order to achieve a steady-state condition, the average current drawn from the electrical network should be equal to the power losses averaged on a time extent $T_{p}$ :

$$
\begin{aligned}
& \Delta E_{g\left[T_{p}\right]}=\Delta E_{a c t\left[T_{p}\right]} \\
& \overline{i_{g}} v_{d c} T_{p}=I_{R E F}^{2} R_{m}\left(t_{f t}+\frac{t_{r}+t_{f}}{3}\right)
\end{aligned}
$$

In order to bound the grid current, the input filter has to be designed to achieve Discontinuous Current Mode (DCM) in the inductor. This operation mode allows changing the amount of grid current averaged in a ripple period of the diode rectifier as a function of the difference between $v_{d c}$ and $v_{g}$, which leads to a voltage-controlled current source without dynamics behaviour for $L_{d c}$. In order to achieve this, $v_{d c}$ must be within the ripple of the rectified voltage and the average grid voltage, i.e. $\overline{v_{g}}<v_{d c}<\hat{v}_{g}$. Figure 7 shows a general block diagram of the developed control system, where two regulation loops are proposed. 


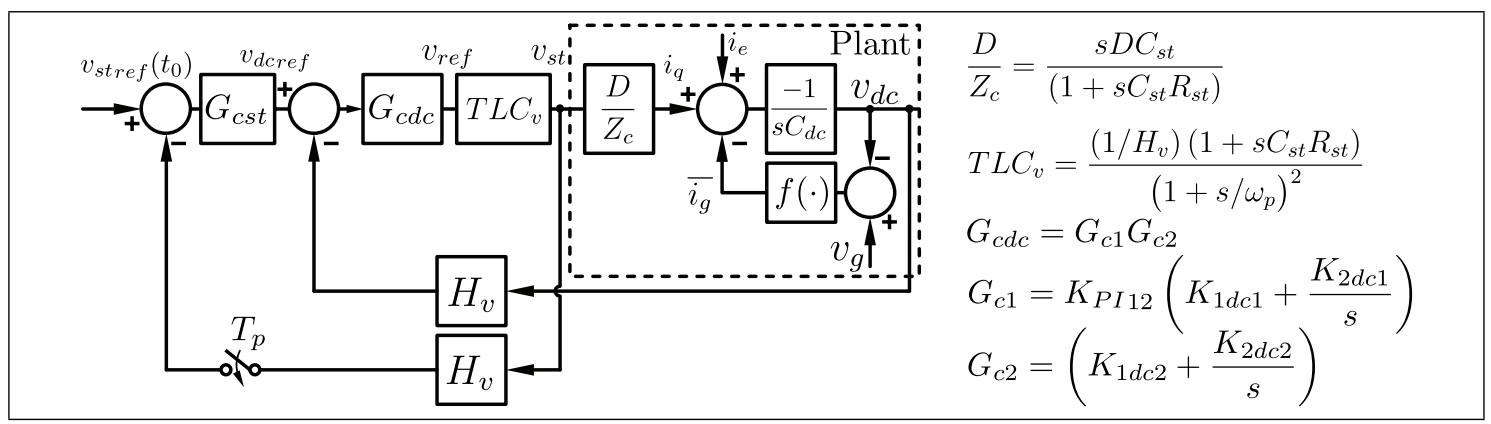

Figure 7: Block diagram of control system for strategy IGCC.

The first one (inner loop) operates during the whole period $T_{p}$ and tries to keep $v_{d c}=v_{d c r e f}$ by changing $v_{s t}(t)$. The second one (outer loop) adjusts the $v_{d c r e f}$ value at the beginning of each pulse bearing in mind two objectives: to obtain an average grid current capable of supplying the power losses, and to obtain a value of $v_{s t}$ at the beginning of each pulse given by design. The latter is particularly important as it defines the minimum voltage for $v_{s t}$ for the next pulse. Regarding plant modelling, it is similar to the case of energy recovery between pulses, except that there is a voltage-controlled current source in the node of $i_{c}, i_{q}$ and $i_{e}$. The expression for the grid current, averaged in an input ripple period, is given by:

$$
\overline{i_{g}}=f\left(v_{d c}, v_{g}, L_{d c}\right) \approx K_{g} v_{d c}+\left(\overline{i_{g_{\max }}}-K_{g} \overline{v_{g}}\right)
$$

where $K_{g}$ is the linearized gain for the current source stage and $\overline{i_{g_{\max }}}$ is the maximum current that can be obtained in DCM mode, which corresponds to $v_{d c}=\bar{v}_{g}$. Concerning the inner loop, the controller is implemented using a double cascaded PI configuration. This controller is designed to obtain a small error in $v_{d c}(t)$ due to $i_{e}(t)$. The outer loop has to set the proper value of $v_{d c r e f}$ that will set the storage element voltage at the beginning of the pulse to a value defined by design. As a consequence, the voltage $v_{s t}$ is sampled at the beginning of each magnet pulse to obtain an error information to adjust $v_{\text {dcref }}$. Then, this outer loop is designed as a discrete controller $G_{c s t}[z]$, sampled to the pulse repetition period.

It must be noticed that $v_{d c}(t)$ has to be adjusted according to (6); then, if any of these parameters change (for instance, in case of grid voltage perturbations or a change in the flat-top current), a transient response of several pulses will be generated due to the limited controllability of the dc-link. This implies a higher exigency on the value of energy to be stored (i.e., the storage element value).

\section{Qualitative waveforms with different strategies control}

In Fig. 8, the most relevant variables (magnet current, grid current and storage voltage) for the different strategies are shown.

\section{Comparative analysis}

In order to make a fair comparison between the developed strategies, some important figures of merit are defined in this section. One of these parameters is the variation of energy in the storage element. This parameter plays a key role in the selection of $C_{s t}$, since the energy gives an idea of the storage element sizing. Concerning this aspect, Table III shows the expressions for the different control strategies, where the term $\Delta E\left[t_{x}\right]$ represents the energy variation in the time interval $t_{x}$. 


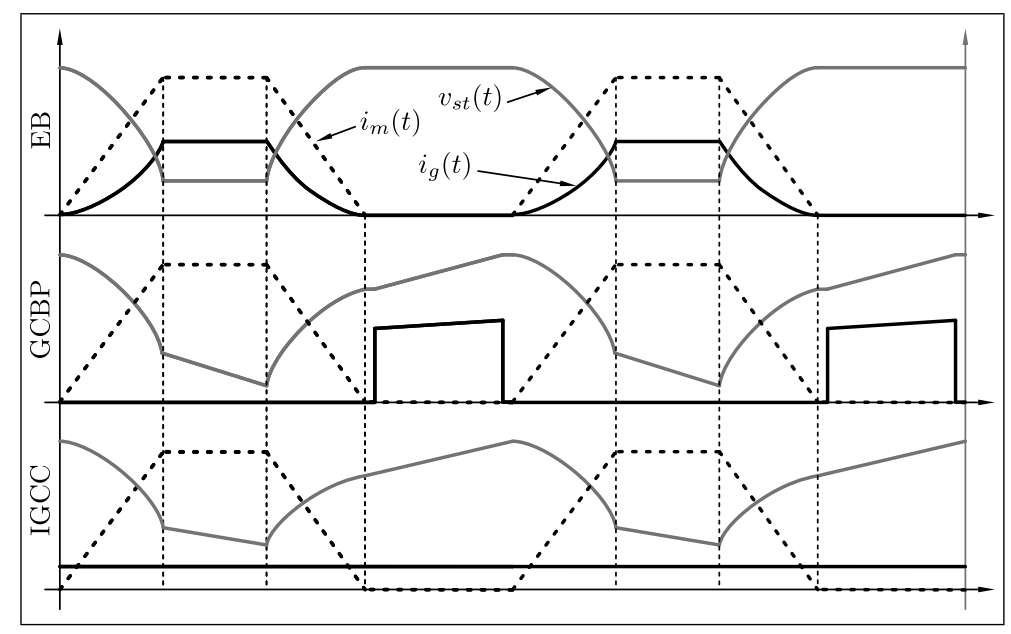

Figure 8: Qualitative waveforms.

In such table, $\Delta E_{s t}$ represents the energy variation in $C_{s t}, \Delta E_{\text {act }}$ represents the active energy variation in the magnet and $\Delta E_{g}$ represents the energy supplied by the grid. In each case, the energy variation is related to the maximum reactive energy in the magnet, $E_{m}=1 / 2 L_{m} I_{r e f}^{2}$.

It can be observed that the maximum energy variation of the storage element in strategy EB corresponds to the maximum reactive energy in the magnet, which is a consequence of the energy balance shown in (2). In strategy GCBP, the energy variation is greater than in the case of strategy EB, since during magnet pulse generation the capacitor $C_{s t}$ must supply both the reactive energy and the active energy. In the case of IGCC, the energy supplied from the electrical grid helps to decrease the energy variation in $C_{s t}$ as shown in the last term of the expression corresponding to this strategy.

Related to the previous, the minimum value of $C_{s t}$ for each strategy is obtained. Such limit value is calculated having into consideration that $C_{s t}$ discharges up to a minimum voltage $v_{s t m i n}$. Table IV shows the expression for $C_{s t}$ using the different strategies.

Regarding the minimum $C_{s t}$ value, it can be noted that in strategy EB this minimum is associated to the maximum reactive energy supplied to the load, which corresponds to the flat-top current. In the case of GCBP, the minimum value results higher than in EB strategy due to the active energy supplied by $C_{s t}$. Hence, the flat-top length is important to define the capacitor. Finally, strategy IGCC allows decrease the capacitor with respect to the one selected in GCBP, due to the energy supplied by the electrical grid during magnet pulse generation.

Another important parameter that was evaluated is the maximum grid current, $i_{g_{\max }}$, as it defines the sizing of the diode bridge rectifier and the rating of the connection on the distribution network. Table $\mathrm{V}$ shows the maximum grid current with each strategy. In each case the maximum grid current is normalized to the current required by the H-bridge during the flat-top time, i.e. $i_{e f t}=\left(I_{R E F}^{2} R_{m}\right) / V_{d c}$.

It can be seen in Table $\mathrm{V}$ that, in the strategy EB, the value of $i_{g_{\max }}$ will depend on the active power in the magnet. In the case of strategy GCBP, the maximum grid current will depend on the relationship between the pulse times and the time between pulses; hence, it can be lower or greater than in the case EB, depending on this relatonship. In such sense, the longer the time between pulses related to the pulse repetition period, the lower the maximum grid current. The IGCC strategy is the most advantageous case, since the current supplied by the grid arises from the active power spread over the pulse period, $T_{p}$. Notice that in all analyzed cases, $i_{g}$ depends on $v_{d c}$ and, hence, on the grid voltage. From the above mentioned, strategy IGCC presents the lower power rating for the input rectifier, since the instantaneous power is equal to the active

Table III: Maximum energy variation in storage capacitor for the different strategies.

\begin{tabular}{l|l}
\hline $\mathrm{EB}$ & $\frac{\Delta E_{s t}\left[t_{r}\right]}{E_{m}}=1$ \\
\hline $\mathrm{GCBP}$ & $\frac{\Delta E_{s t}\left[t_{r}+t_{f t}\right]}{E_{m}}=1+\frac{\Delta E_{a c t}\left[t_{r}+t_{f t}\right]}{E_{m}}$ \\
\hline IGCC & $\frac{\Delta E_{s t}\left[t_{r}+t_{f t}\right]}{E_{m}}=1+\frac{\Delta E_{a c t}\left[t_{r}+t_{f t}\right]}{E_{m}}-\frac{\Delta E_{g}\left[t_{r}+t_{f t}\right]}{E_{m}}$ \\
\hline
\end{tabular}


Table IV: Minimum $C_{s t}$ for the different strategies.

\begin{tabular}{l|l}
\hline $\mathrm{EB}$ & $C_{s t}>\frac{2 E_{m}}{v_{s t}^{2}(0)-v_{s t_{\text {min }}^{2}}}$ \\
\hline $\mathrm{GCBP}$ & $C_{s t}>\frac{2 E_{m}}{v_{s t}^{2}(0)-v_{s t_{\text {min }}}^{2}}\left[1+2 \frac{R_{m}}{L_{m}}\left(t_{f t}+\frac{t_{r}}{3}\right)\right]$ \\
\hline $\mathrm{IGCC}$ & $C_{s t}>\frac{2 E_{m}}{v_{s t}^{2}(0)-v_{s t_{\text {min }}}^{2}}\left[1+2 \frac{R_{m}}{L_{m}}\left(t_{f t}+\frac{t_{r}}{3}\right)-\frac{\overline{i_{g}} v_{d c}\left(t_{r}+t_{f t}\right)}{E_{m}}\right]$ \\
\hline
\end{tabular}

Table V: Maximum grid current for the different strategies.

\begin{tabular}{l|l}
\hline $\mathrm{EB}$ & $\frac{i_{g_{\max }}}{i_{e f t}}=1$ \\
\hline $\mathrm{GCBP}$ & $\frac{i_{g_{\max }}}{i_{e f t}} \geq 2 \frac{\left(t_{f t}+\left(t_{r}+t_{f}\right) / 3\right)}{T_{p}-\left(t_{r}+t_{f t}+t_{f}\right)}$ \\
\hline $\mathrm{IGCC}$ & $\frac{i_{g_{\max }}}{i_{\text {eft }}}=\frac{\left(t_{f t}+\left(t_{r}+t_{f}\right) / 3\right)}{T_{p}}$ \\
\hline
\end{tabular}

Table VI: Parameters of the implemented controllers.

\begin{tabular}{|c|c||c|c||c|c|}
\hline \multicolumn{2}{|c||}{ Coefficients of $T L C_{v}(s)$} & \multicolumn{2}{c||}{ Coefficients of $G_{c d c 1}(s), G_{c d c 2}(s)$} & \multicolumn{2}{c|}{ Coefficients of $G_{c s t}[z]$} \\
\hline \hline$K_{c}$ & 10 & $k_{P I 12}$ & $-3.44 \cdot 10^{-2}$ & $k_{c 1}$ & 0.85 \\
\hline$k_{v S F}$ & $1.53 \cdot 10^{-2}$ & $k_{1 d c 1}$ & 1 & $k_{c 2}$ & $-8.93 \cdot 10^{-5}$ \\
\hline$k_{i S F}$ & $9.08 \cdot 10^{-4}$ & $k_{2 d c 1}$ & 50.27 & & \\
\hline$k_{1 S F}$ & $1.81 \cdot 10^{2}$ & $k_{1 d c 2}$ & $1 \cdot 10^{-2}$ & & \\
\hline$k_{2 S F}$ & $1.26 \cdot 10^{4}$ & $k_{2 d c 2}$ & 502.7 & & \\
\hline
\end{tabular}

average power. Concerning the $2 \mathrm{Q}$ converter, strategy GCBP is the most demanding one in terms of instantaneous power, since the $2 \mathrm{Q}$ converter has to handle the full load power, while $\mathrm{EB}$ is the less demanding case since the $2 \mathrm{Q}$ only has to handle the instantaneous reactive power.

\section{Simulations}

In order to evaluate the performance of the strategies proposed, simulation experiments using the parameters of Table I were conducted. In this case, a voltage variation in the storage element, $\Delta V_{s t}$, close to $300 \mathrm{~V}$, a voltage at the beginning of the pulse of $v_{s t}\left(t_{0}\right)=650 \mathrm{~V}$ and a grid voltage $\overline{v_{g}}=700 \mathrm{~V}$ (applied through a $50 \mathrm{~Hz}$ transformer at the input of a six-pulse diode rectifier) were selected. Using the expressions in Table IV, the capacitor value for strategy EB will be around $C_{s t}=200 \mathrm{mF}$, for strategy $\mathrm{GCBP}$ it is close to $C_{s t}=250 \mathrm{mF}$ and for strategy IGCC should be close to $220 \mathrm{mF}$. However, the value obtained for strategy IGCC only accounts for steady-state operation. Therefore, in order to take into consideration a transient condition in which the average current from the grid is lower than the required one, $C_{s t}=250 \mathrm{mF}$ is chosen. Notice that, as shown in Table IV, the capacitor value in strategies GCBP and IGCC strongly depends on the pulse parameters $\left(t_{r}, t_{f t}\right)$, while in strategy EB it depends on the maximum reactive energy. Finally, the H-bridge current during flat-top time is $i_{e f t}=(450 \mathrm{~A})^{2} 0.4 \Omega / 700 \mathrm{~V}=115 \mathrm{~A}$. Table VI shows the parameters of the controllers used in each strategy. Figure 9(a) shows the simulation results for the current demanded by the H-bridge $i_{e}(t)$, grid current $i_{g}(t)$ and a filtered version of the energy storing element current, $i_{q}(t)$. It can be noticed that in the three cases the grid current is bounded to the H-bridge current during flat-top time: $i_{g}{ }^{E B}=115 \mathrm{~A}$, $i_{g}{ }^{G C B P} \approx 80 \mathrm{~A}$ and $i_{g}{ }^{I G C C} \approx 23 \mathrm{~A}$. Figure $9(\mathrm{~b})$ shows the simulation results for the voltages $v_{s t}(t), v_{g}(t)$ and $v_{d c}$. Notice the variation in the grid voltage as a function of the grid current, due to the leakage inductance and resistance of the transformer used. In this sense, the minimum perturbation on $v_{d c}(t)$ occurs in strategy IGCC, due to the low current supplied. Concerning storage element voltage, it can be seen that in strategy EB the minimum voltage is reached at the end of the rise time, while in the remaining strategies $C_{s t}$ continues its discharge up to the end of flat-top time. In EB and GCBP the maximum voltage variation adopted is achieved, while in IGCC the voltage variation is lower, since a higher value for $C_{s t}$ has to be used to take into account the transient response to a change in any of the parameters that define $\overline{i_{g}}$ in this control scheme. 


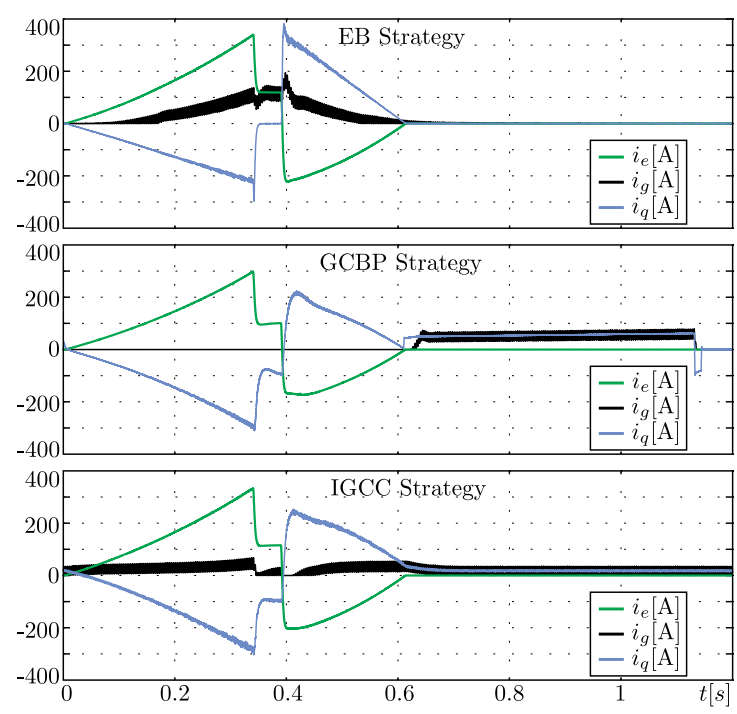

(a) Current demanded by the H-bridge $\left(i_{e}(t)\right)$, grid current $\left(i_{g}(t)\right)$ and 2-Q averaged current $\left(i_{q}(t)\right)$.

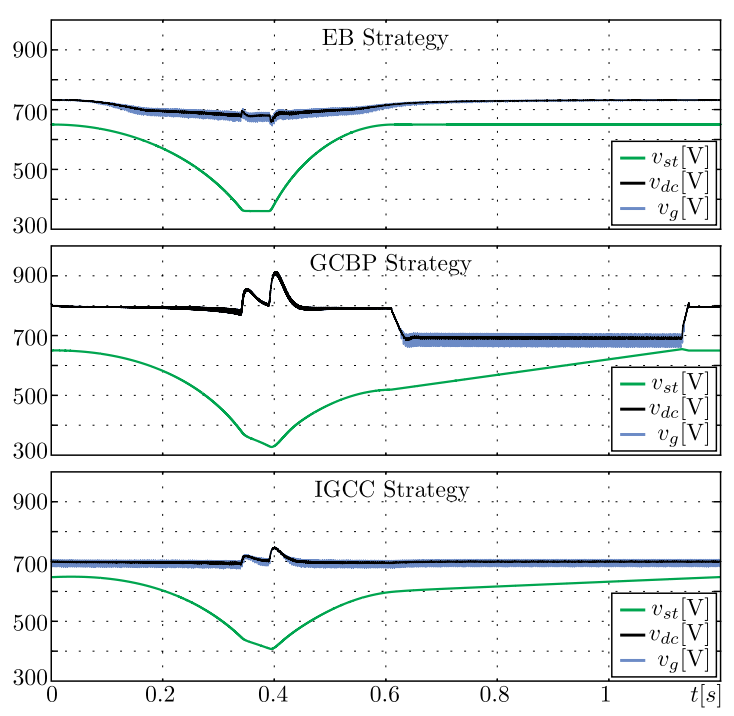

(b) Voltage in the storage element $\left(v_{s t}(t)\right)$, dc-link voltage $\left(v_{d c}(t)\right)$, rectified grid voltage $\left(v_{g}(t)\right)$.

Figure 9: Simulation of a magnet current cycle from 0 to $450 \mathrm{~A}$.

From the results, it can be seen that IGCC results the best strategy, since it presents a lower, constant input current, which implies a low perturbation on the grid and an input module with lower power rating. However, it should be noticed that the analysis was performed considering a grid without perturbation and a steady-state condition in the pulse parameters.

\section{Conclusions}

In this work, a topology based on a two-quadrant (2Q) regulator connected to the DC link of a 4-quadrant magnet supply was analyzed. A comparative study between three strategies was performed, with the aim of defining several aspects of the system that results interesting for the power converter design. Analytical calculations and simulation results indicate that IGCC achieve the lower current from the electrical network; however, this strategy presents a high sensitivity to grid voltage fluctuations and changes in pulse parameters. This leads to an increase in the storage capacitance. In the EB strategy, the energy supplied by the electrical network during magnet current pulse generation is the higher, which leads to the higher current and the lower capacitance. Finally, the GCBP presents a maximum current that depends on the time between pulses; for the analyzed pulse parameters, this current has resulted comparable to the IGCC strategy. With respect to capacitance, GCBP presents the maximum capacitance value, since the grid does not supplies energy during magnet pulse generation.

\section{References}

[1] "Power Converter Topologies with Energy Recovery and Grid Power Limitation for Inductive Load Applications," submited to 17th Internacional European Power Electronics Conference and Applications, EPE15, Sep. 2015.

[2] S. Vesti, T. Suntio, J. A. Oliver, R. Prieto, and J. A. Cobos, "Impedance-Based Stability and Transient-Performance Assessment Applying Maximum Peak Criteria," IEEE Transactions on Power Electronics, vol. 28, no. 5, pp. 2099-2104, May 2013.

[3] B. Choi, D. Kim, D. Lee, S. Choi, and J. Sun, "Analysis of Input Filter Interactions in Switching Power Converters," IEEE Transactions on Power Electronics, vol. 22, no. 2, pp. 452-460, Mar. 2007.

[4] J. Sun, "Impedance-Based Stability Criterion for Grid-Connected Inverters," IEEE Transactions on Power Electronics, vol. 26, no. 11, pp. 3075-3078, Nov. 2011.

[5] C. Liu, A. Ridenour, and J.-S. Lai, "Modeling and Control of a Novel Six-Leg Three-Phase HighPower Converter for Low Voltage Fuel Cell Applications," IEEE Transactions on Power Electronics, vol. 21, no. 5, pp. 1292-1300, Sep. 2006.

[6] T. Pavlovic, T. Bjazic, and Z. Ban, "Simplified Averaged Models of DCDC Power Converters Suitable for Controller Design and Microgrid Simulation," IEEE Transactions on Power Electronics, vol. 28 , no. 7 , pp. 3266-3275, Jul. 2013. 